\title{
Unmoderated Posters: Oncology - Penis, Testis and Urethra
}

\section{UP-12}

The Anatomy of Post-chemotherapy Retroperitoneal Lymph Node Dissections for Testis Cancer: A Cadaveric Study of the Aortic Plexus and Patterns of Lumbar Vessels

Beveridge, Tyler S. ${ }^{1}$; Johnson, Marjorie ${ }^{1}$; Power, Adam²; Allman, Brian L. ${ }^{1}$; Power, Nicholas ${ }^{2}$

${ }^{1}$ Western University, London, ON, Canada; ${ }^{2}$ London Health Sciences Centre, London, ON, Canada

Introduction and Objectives: Post-chemotherapy retroperitoneal lymph node dissection (pc-RPLND) for testis cancer requires an expert knowledge of retroperitoneal anatomy. The presence of aberrant lumbar vessels may lead to intraoperative complications, and damage to the aortic plexus often results in retrograde ejaculation. Despite attempts to minimize complications using unilateral template dissections, there has never been a robust anatomical description of the retroperitoneum. The aim of this study is to determine the variability in the lumbar vessels and to map the nerves of the aortic plexus for preservation during surgery.

Methods: Anatomy of the lumbar vessels was determined by embalmed cadaveric dissections $(n=25)$ and the aortic plexus was mapped in fresh, frozen human cadavers $(n=7)$. Using standard histochemical procedures, ganglia of the aortic plexus were verified with H\&E staining while anti-tyrosine hydroxylase staining confirmed the presence of sympathetic neurons. Results: Vascular dissections revealed a left-side dominance of lumbar veins that were not equally-spaced; rather, the inter-vein distance progressively increased for the more inferior veins. In contrast, the lumbar arteries were bilaterally symmetrical with consistent inter-artery distance along the aorta. All aortic plexus dissections revealed 3 previously identified ganglia (right/ left spermatic ganglia and inferior mesenteric ganglion), and one newly discovered ganglion; all of which were supplied by the L1 and L2 lumbar splanchnic nerves.

Conclusions: Our anatomical description of the lumbar vessels and the nerves of the aortic plexus may prove useful for surgeons performing pcRPLND. We observed unique patterns in the lumbar vasculature, and identified peripheral nerves within the aortic plexus which we hypothesize contain fibers controlling ejaculation. Finally, the newly discovered ganglion may be integral in preserving fertility in males undergoing pc-RPLND. 